Mar. Drugs 2006, 4, 15-21

Marine Drugs

ISSN 1660-3397

(C) 2006 by MDPI

www.mdpi.org/marinedrugs

Full Research Article

\title{
Effects of Lissoclibadins and Lissoclinotoxins, Isolated from a Tropical Ascidian Lissoclinum cf. badium, on IL-8 production in a PMA-stimulated Promyelocytic Leukemia Cell Line
}

\section{Taiko Oda $^{1{ }^{1} *}$, Takeshi Fujiwara ${ }^{2}$, Hongwei Liu ${ }^{2, \dagger}$, Kazuyo Ukai ${ }^{2}$, Remy E. P. Mangindaan ${ }^{3}$,} Masataka Mochizuki ${ }^{1}$ and Michio Namikoshi ${ }^{2}$

1 Department of Pharmaceutical Sciences, Kyoritsu University of Pharmacy, Shibakoen, Minato-ku, Tokyo 105-8512, Japan

2 Department of Ocean Sciences, Tokyo University of Marine Science and Technology, Minato-ku, Tokyo 108-8477, Japan

3 Faculty of Fisheries and Marine Science, Sam Ratulangi University, Kampus Bahu, Manado 95115, Indonesia

* Author to whom correspondence should be addressed: Tel. +81-3-5400-2497, Fax +81-3-5400-2497, E-mail: oda-ti@kyoritsu-ph.ac.jp

${ }^{\dagger}$ Present address: Tsinghua University, Tsinghua Campus, The University Town, Shenzhen, Peoples Republic of China

Received: 28 December 2005 / Accepted: 17 February 2006 / Published: 21 February 2006

\begin{abstract}
The effects of seven compounds 1-7, isolated from a tropical ascidian Lissoclinum cf. badium, on IL-8 production in PMA-stimulated HL-60 cells were examined. Lissoclibadins 2 (2) and 3 (3) and lissoclinotoxin F (5) increased the IL-8 production in a dose-dependent manner. Compounds 2 and $\mathbf{5}$ are structural isomers possessing dimeric structures of trans and cis-orientations, respectively, and showed a very similar activity on the induction of IL-8 levels. Compound 3 and lissoclinotoxin E (4) are also structural isomers having dimeric trans and cis-structures, respectively, but $\mathbf{4}$ did not induce the IL-8 production. Lissoclibadin 1 (1, trimeric compound) and two monomeric compounds (6 and 7) did not increase the IL-8 level. Therefore, the differences in their structures remarkably affected the IL-8 production activity, the inhibition of cell proliferation, and the survival of HL-60 cells. Lissoclibadin 2 was the most interesting compound of the seven metabolites tested in this study.
\end{abstract}

Keywords: Tropical ascidian, Lissoclinum cf. badium, Lissoclibadins, Lissoclinotoxins, IL8, HL-60 cells. 


\section{Introduction}

Ascidians are prolific sources of new chemical entities with unique structures and potent biological activities [1,2]. In the course of our studies on bioactive metabolites of marine organisms, we have reported the isolation and structures of three new compounds, lissoclibadins 1 (1), 2 (2) and 3 (3), together with four known compounds (4-7) from a tropical ascidian Lissoclinum cf. badium. The three new compounds have polysulfur aromatic amine structures and inhibited the growth of the human promyelocytic leukemia cell line HL-60 [3,4]. Compounds 1-7 also showed growth inhibitory activity against Chinese hamster V79 cells, but none of the seven compounds inhibited an assembly of microtubule proteins in vitro (unpublished data). Although, in our experiments, the relationship between the inhibition of microtubule assembly and of the colony formation of V79 cells has been observed for several compounds [5], compounds 1-7 did not show a correlation between the two bioactivities.

In this study, we attempted to show the effects of these compounds (1-7) on the production of an inflammatory cytokine, interleukin-8 (IL-8). IL-8 is a member of the superfamily of C-X-C chemokines and a chemotactic factor for $\mathrm{T}$ cells, neutrophils and basophils [6]. IL-8 expression has been detected in a variety of human cancers and is suggested to be a factor in tumor progression and metastasis [7-10]. Therefore, the regulation of IL-8 production is an important medical problem.

Three compounds (2, 3 and 5) increased the IL-8 production in PMA-stimulated HL-60 cells, but the other four compounds were not active at $10 \mu \mathrm{M}$. These compounds showed four patterns of activity against HL-60 cells on the IL-8 production, inhibition of the cell proliferation and cytotoxicity. These patterns did not correlate with their structures.

\section{Materials and Methods}

\subsection{Materials}

Lissoclibadins 1 (1), 2 (2) and 3 (3), lissoclinotoxins E (4) and F (5), 3,4-dimethoxy-6-(2'- $N, N$ dimethylaminoethyl)-5-(methylthio)benzotrithiane (6) and $N, N$-dimethyl-5-(methylthio)varacin (7) were isolated from the ascidian $L$. cf. badium collected in Indonesia, as described previously [4]. The structures of seven compounds are shown in Figure 1. Dimethylsulfoxide (DMSO) was purchased from Pierce Chemical Co. (Rockfield, IL) and fetal bovine serum (FBS) was obtained from GIBCO after checking of the lot. All other reagents and chemicals used were of the highest grade available commercially.

\subsection{Cell lines and culture conditions}

The HL-60 cell line was obtained from the Japanese Cancer Research Resources Bank (JCRB, Kamiyoga, Tokyo, Japan). This cell line was maintained in tissue culture dishes in RPMI 1640 medium (Nissui Seiyaku, Tokyo, Japan), supplemented with 10\% heat-inactivated fetal calf serum (FCS), $2 \mathrm{mM}$ glutamine, $100 \mathrm{U} / \mathrm{ml}$ of penicillin $\mathrm{G}$ and $100 \mu \mathrm{g} / \mathrm{ml}$ of streptomycin. 


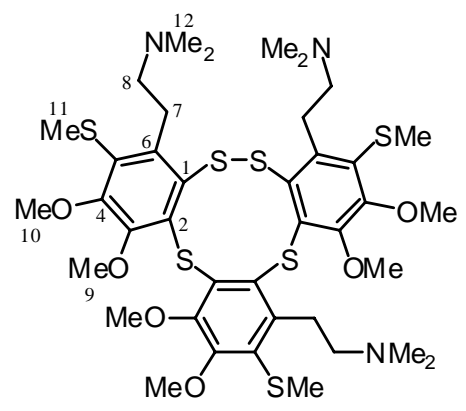

Lissoclibadin 1 (1)

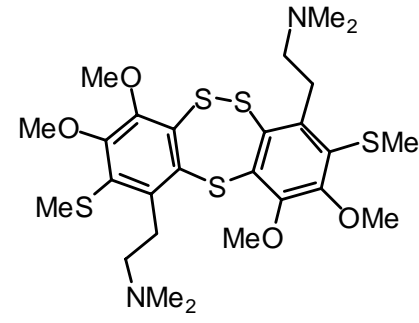

Lissoclibadin 2 (2)

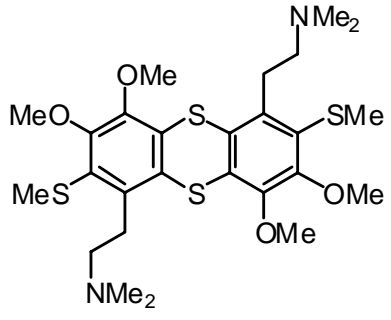

Lissoclibadin 3 (3)

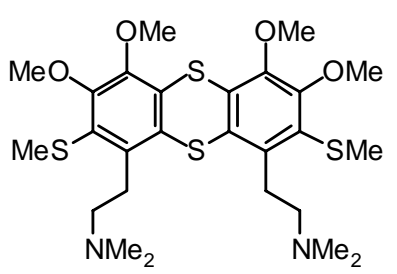

Lissoclinotoxin E (4)

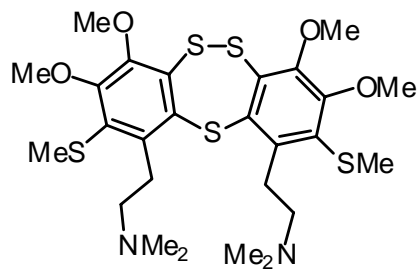

Lissoclinotoxin F (5)<smiles></smiles>

6<smiles>COc1c(OC)c(OC)c2ssssc2c1CCN(C)C</smiles>

7

Figure 1. Structures of compounds 1-7.

\subsection{Detection of human IL-8 by ELISA}

The IL-8 concentrations of the culture supernatants under control and various test conditions were measured by ELISA using a combination of monoclonal and polyclonal antibodies [11]. All samples were assayed at least in duplicate. Data are presented as the mean \pm SE of three independent experiments.

\subsection{Determination of cell proliferation}

Cell proliferation was evaluated by enumerating the viable cells using the MTT formazan production method [12]. HL-60 cells $\left(1 \times 10^{6}\right.$ cells/ml) were treated with PMA (with or without test compounds) and then transferred to 96-well microtiter plates. After incubation for $24 \mathrm{~h}, 20 \mu \mathrm{l}$ of MTT reagent (5 mg/ml in PBS) was added to each well and further incubated for $3 \mathrm{~h}$. The production of formazan was assessed by measuring the optical density $\left(\mathrm{OD}_{570} \mathrm{~nm}\right)$. Data are shown as values relative (\%) to each PMA-stimulated optical density.

\subsection{Determination of cytotoxicity}

The lethality of each compound was estimated by measuring the release of lactate dehydrogenase (LDH) [13]. Data are shown as values relative (\%) to the release of LDH from all cells at each optical density. 

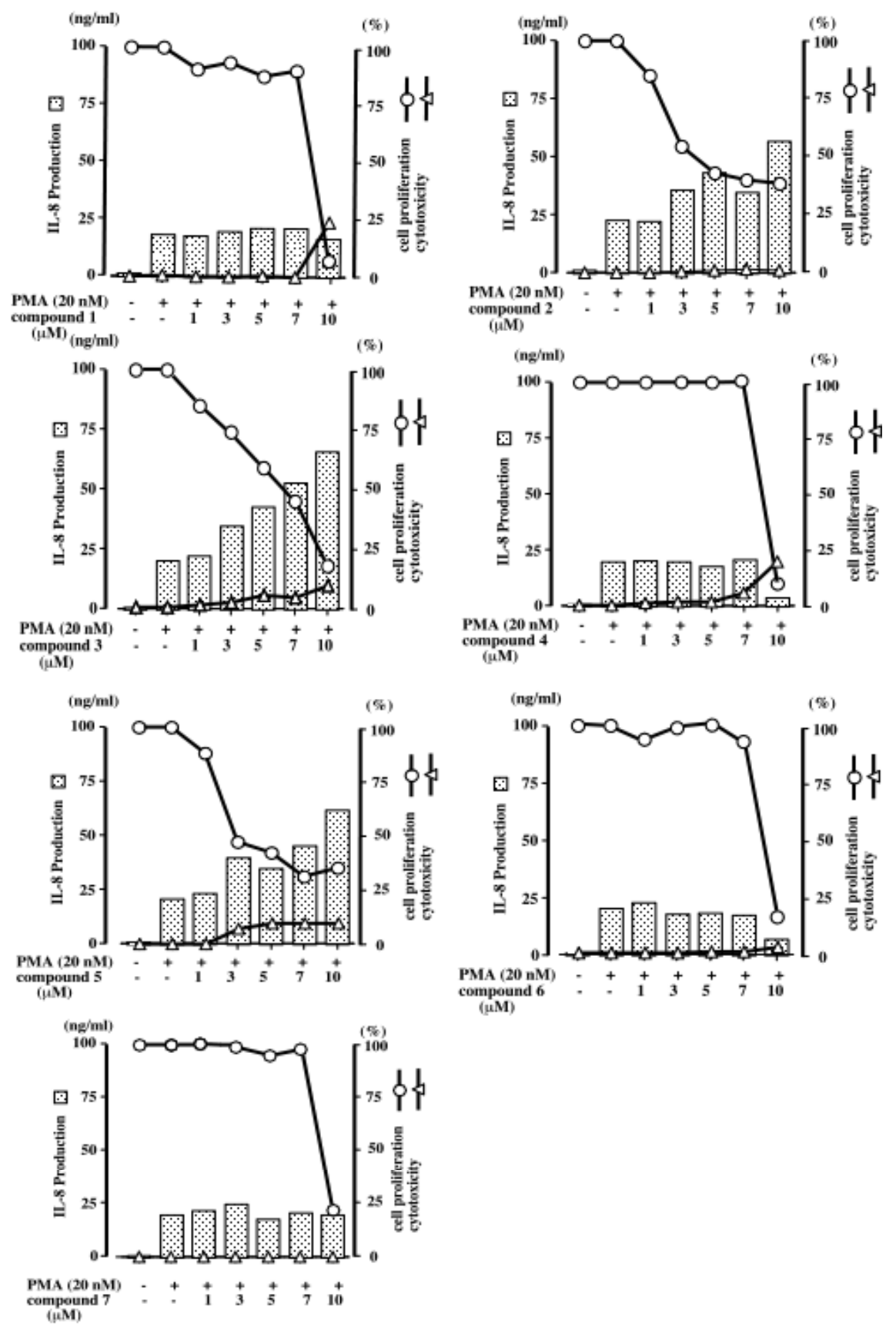
(uM)

Figure 2. Effects of compounds 1-7 on IL-8 production, cell proliferation and cytotoxicity in PMA-stimulated HL-60 cells. Seven panels show the effects of compounds 1-7 on IL-8 production, cell proliferation and cytotoxicity after the addition of PMA (20 nM), respectively. HL-60 cells $\left(1 \times 10^{6}\right.$ cells $\left./ \mathrm{ml}\right)$ were treated with PMA $(20 \mathrm{nM})$ and the indicated concentration of each compound for $24 \mathrm{~h}$. The IL-8 concentration in the culture supernatant under each condition was determined by ELISA, as described in the Materials and Methods. Data are the mean values of three independent experiments. Cell proliferation and cytotoxicity are described in the Materials and Methods. 


\section{Results}

\subsection{Effects of compounds 1-7 on IL-8 production by PMA-stimulated HL-60 cells}

The effects of compounds 1-7 on the IL-8 production were examined using HL-60 cells stimulated with $20 \mathrm{nM}$ of PMA. The results are shown in Figure 2. The production of IL-8 was increased by compounds 2, 3 and 5 from HL-60 cells under PMA stimulation at higher concentrations (3-10 $\mu \mathrm{M})$. However, the other four compounds (1, 4, 6 and 7) did not induce an IL-8 production under the same experimental conditions.

\subsection{Inhibition of cell proliferation and cytotoxicity of compounds 1-7 against HL-60 cells}

The cell proliferation and cytotoxicity of the IL-8 production by compounds 1-7 were determined using the MTT and LDH methods. The results are shown in Figure 2. Compounds 2, $\mathbf{3}$ and $\mathbf{5}$ inhibited the cell proliferation of HL-60 cells in a dose-dependent manner (20-90\%) at 1-10 $\mu \mathrm{M}$. Compounds 1 and $\mathbf{4}$ showed high cytotoxicity at $10 \mu \mathrm{M}$, and moderate cytotoxicity was observed with compounds 3 and 5 at $5-10 \mu \mathrm{M}$.

\section{Discussion}

The effects of seven compounds (1-7) against HL-60 cells on the IL-8 production, inhibition of the cell proliferation, and cytotoxicity are summarized in four patterns. The most interesting pattern was observed for lissoclibadin 2 (2). Compound 2 stopped the proliferation of HL-60 cells and induced the IL-8 production in a dose-dependent manner (Figure 2). Therefore, the IL-8 production correlated with the cell proliferation in this case. Moreover, compound $\mathbf{2}$ did not show cytotoxicity, even at a concentration of $10 \mu \mathrm{M}$. Compound 2 showed a characteristic profile of the in vitro growth inhibitory activity against 12 cancer cell lines (unpublished data). Therefore, lissoclibadin 2 (2) is the most interesting compound in the seven metabolites from $L$. cf. badium tested.

The effects of compounds $\mathbf{3}$ and $\mathbf{5}$ are categorized in the second pattern, which showed the inhibition of proliferation and induction of the IL-8 production in a dose-dependent manner to be similar to the effects of $\mathbf{2}$ (Figure 2). Therefore, the IL-8 production also correlated with the cell proliferation in this case. However, compounds $\mathbf{3}$ and $\mathbf{5}$ were moderately cytotoxic at higher concentrations $(5-10 \mu \mathrm{M})$.

The third and forth patterns showed similar activity on the cell proliferation and IL-8 production. Compounds 1 and 4, the third pattern, and compounds 6 and 7, the forth pattern, did not induce the production of IL-8 and strongly inhibited the proliferation at $10 \mu \mathrm{M}$ (Figure 2). The high cytotoxicity was detected for compounds $\mathbf{1}$ and $\mathbf{4}$ at $10 \mu \mathrm{M}$, while compounds $\mathbf{6}$ and $\mathbf{7}$ were not cytotoxic.

Compounds $\mathbf{2}$ and $\mathbf{5}$ are structural isomers (Figure 1) and revealed an activity very similar to each other on the IL-8 production and cell proliferation, although compound $\mathbf{5}$ showed moderate cytotoxicity against HL-60 cells (Figure 2). The orientation of the two aromatic amine units in compounds 2 and 5 did not affect their activity. 
Although compounds $\mathbf{3}$ and $\mathbf{4}$ are also structural isomers (Figure 1), they had different activities on the IL-8 production and cell proliferation (Figure 2). Therefore, in this case, the orientation of two units is critical for their activity.

Compound 4 showed the same profile of activity on the IL-8 production, cell proliferation, and cytotoxicity as that of compound 1, which has a trimeric structure. Two monomeric compounds, 6 and 7, revealed a very similar profile of activity to those of compounds $\mathbf{1}$ and $\mathbf{4}$ (Figure 2). A slight difference was detected in their cytotoxicity at $10 \mu \mathrm{M}$. The structures of these four compounds are very different to each other.

Patil et al. reported the isolation of lissoclin disulfoxide from the South African ascidian Lissoclinum sp. as the inhibitor of IL-8R $\alpha$ and IL-8R $\beta$ receptors [14]. ${ }^{1} \mathrm{H}$ and ${ }^{13} \mathrm{C}$ NMR data for lissoclin disulfoxide were identical to those for lissoclinotoxin F (5). Compounds 2 and 3 may have a similar inhibitory activity against IL-8 receptors.

From the results obtained in this study, it was hard to deduce a correlation between the structures of seven compounds and the activity against HL-60 cells on the IL-8 production, cell proliferation and cytotoxicity. Investigations of structure-activity relationships and target molecule(s) of these compounds are interesting subjects for future study.

\section{Acknowledgements}

We thank Prof. Tadashi Kasahara for providing the IL-8 monoclonal and polyclonal antibodies. This work was supported in part by a Grant-in-Aid for Scientific Research on Priority Areas 17035029 from The Ministry of Education, Culture, Sports, Science and Technology (MEXT) of Japan to M. N.

\section{References}

1. Blunt, J. W.; Copp, B. R.; Munro, M. H. G.; Northcote, P. T.; Prinsep, M. R. Marine natural products. Nat. Prod. Rep., 2005, 22, 15-61; 2004, 21, 1-49; 2003, 20, 1-48.

2. Faulkner, D. J. Marine natural products. Nat. Prod. Rep., 2002, 19, 1-48; 2001, 18, 1-49; 2000, 17, 7-55; 1999, 16, 155-198; 1998, 15, 113-158; 1997, 14, 259-302; 1996, 13, 75-125; 1995, 12 , 223-269; 1994, 11, 355-394; 1993, 10, 497-539; 1992, 9, 323-364; 1991, 8, 97-147; 1990, 7 , 269-309; 1988, 5, 613-576; 1987, 4, 539-576; 1986, 3, 1-33; 1984, 1, 251-280, 551-598.

3. Liu, H.; Pratasik, S. B.; Nishikawa, T.; Shida, T.; Tachibana, K.; Fujiwara, T.; Nagai, H.; Kobayashi, H.; Namikoshi, M. Lissoclibadin 1, a Novel Trimeric Sulfur-Bridged Dopamine Derivative, from the Tropical Ascidian Lissoclinum cf. badiu. Tetrahedron Lett. 2004, 45, 70157017.

4. Liu, H.; Fujiwara, T.; Nishikawa, T.; Mishima, Y.; Nagai, N.; Shida, T.; Tachibana, K.; Kobayashi, H.; Mangindaan, R. E. P.; Namikoshi, M. Lissoclibadins 1-3, three new polysulfur alkaloids, from the ascidian Lissoclinum cf. badium. Tetrahedron 2005, 61, 8611-8615.

5. Oda, T.; Sakakibara, Y,; Sato, Y,; Hanzawa, H,; Hata, T. Effects of (+)-, (-)- and (+-)-Indenestrols A and B on Microtubule Polymerization. Chem. Pharm. Bull. 1992, 40, 588-592.

6. Graves, D. T.; Jiang, Y. Molecular cloning and functional analysis of the promoter of the human squalene synthase gene. Crit. Rev. Oral Biol. Med. 1995, 6, 109-118. 
7. Di Celle, P. F.; Carbone, A.; Marchis, D.; Zhou, D.; Sozzani, S.; Zupo, S.; Pini, M.; Mantovani, A.; Foa, R.; Cytokine gene expression in B-cell chronic lymphocytic leukemia: evidence of constitutive interleukin-8 (IL-8) mRNA expression and secretion of biologically active IL-8 protein. Blood 1994, 84, 220-228.

8. Green, A. R.; Green, V. L.; White, M. C.; Speirs, V. Expression of Cytokine messenger RNA in normal and neoplastic human breast tissue: identification of interleukin-8 as a potential regulatory factor in breast tumours. Int. J. Cancer 1997, 72, 937-941.

9. Konig, B.; Steinbach, F.; Janocha, B.; Drynda, A.; Stumm, M.; Philipp, C.; Allhoff, E. P.; Konig, W. The differential expression of proinflammatory cytokines IL-6, IL-8 and TNF-alpha in renal cell carcinoma. Anticancer Res. 1999, 19, 1519-1524.

10. Galffy, G.; Mohammed, K. A.; Dowling, P. A.; Nasreen, N.; Ward, M. J. Antony, V. B. Interleukin 8: an autocrine growth factor for malignant mesothelioma. Cancer Res. 1999, 59, 367-371.

11. Kasahara, T.; Oda, T.; Hatake, K.; Akiyama, M.; Mukaida, N.; Matsushima, K. Interleukin-8 and monocyte chemotactic protein-1 production by a human glioblastoma cell line, T98G in coculture with monocytes: involvement of monocyte-derived interleukin-1alpha. Eur. Cytokine Netw. 1998, 9, 47-55.

12. Carmichael, J.; DeGraff, W. G.; Gazdar, A. F.; Minna, J. D.; Mitchell, J. B. Evaluation of a tetrazolium-based semiautomated colorimetric assay: assessment of chemosensitivity testing. Cancer Res. 1987, 47, 939-942.

13. Shimizu, S.; Nomoto, M.; Naito, S.; Yamamoto, T.; Momose, K. Stimulation of nitric oxide synthase during oxidative endothelial cell injury. Biochem. Pharmacol. 1998, 55, 77-83.

14. Patil, A. D.; Freyer, A. J.; Killmer, L.; Zuber, G.; Carte, B.; Jurewicz, A. J.; Johnson, R. K. Lissoclin disulfoxide, a novel dimeric alkaloid from the ascidian Lissoclinum sp.: inhibitor of interleukin-8 receptors. Nat. Prod. Lett. 1997, 10, 225-229.

Sample availability: Not available.

(C) 2006 by MDPI (http://www.mdpi.org). Reproduction is permitted for noncommercial purposes. 